\title{
Magnetoplasmons in a High Electron Mobility GaAs/AlGaAs Heterostructure
}

\author{
M. Bialek*, K. Karpierz, B. Pietka, M. Grynberg, J. Łusakowski \\ Institute of Experimental Physics, Faculty of Physics, University of Warsaw, Hoża 69, 00-681 Warsaw, Poland \\ M. Czapkiewicz, K. Fronc, J. Wróbel \\ Institute of Physics, PAS, al. Lotników 32/46, 02-668 Warsaw, Poland \\ AND V. UMANSKY \\ Weizmann Institute of Science, Rehevot 76100, Israel
}

\begin{abstract}
We have observed a multimode spectrum of magnetoplasmons in the Hall bars processed on a high electron mobility GaAs/AlGaAs heterostructure. We have found that the dispersion relation of these excitation follows square root dependence. Calculated wavelength of the fundamental magnetoplasmon mode fits to the width of sample.
\end{abstract}

PACS: 73.20.Mf

\section{Introduction}

The frequency $\omega$ of a plasmon in a magnetic field (i.e., a magnetoplasmon) is governed by the relation [1]:

$$
\omega^{2}=\omega_{\mathrm{p}}^{2}+\omega_{\mathrm{c}}^{2}
$$

where $\omega_{\mathrm{p}}$ is a frequency of the plasmon in zero magnetic field, $\omega_{\mathrm{c}}=e B / m^{*}$ is the cyclotron frequency, $e$ is the elementary charge, $B$ is a magnetic field and $m^{*}$ is the effective mass of electron. The plasmon frequency in two-dimensional systems depends on its wave vector. To the first approximation, this dependence is square-like

$$
\omega_{\mathrm{p}}^{2}=\frac{n e^{2} k}{2 m^{*} \epsilon_{\mathrm{eff}} \epsilon_{0}},
$$

where $n$ is the electron concentration and $k$ is the plasmon wave vector [2]. If one considers the fundamental and a series of higher plasmon modes, one gets a picture shown in Fig. 1 where the evolution of magnetoplasmon frequency is plotted as a function of magnetic field.

An important factor in the dispersion relation, Eq. (2), is the dielectric function $\epsilon_{\text {eff }}$. In a heterostructure where a two-dimensional electron gas (2DEG) is not screened with a metallic gate electrode (this is the case considered in the present paper), one gets $\epsilon_{\text {eff }}=\left[\epsilon_{1}+\epsilon_{2} \tanh (k D)\right] / 2$, where $D$ is thickness of the barrier, $\epsilon_{1}$ is a dielectric constant of the channel material and $\epsilon_{2}$ is dielectric constant of a barrier material ( $\mathrm{GaAs}$ and GaAlAs in our case, respectively) [3]. In case of $k D \ll 1$ (long plasmon wavelength comparing to $D)$, the $\epsilon_{\text {eff }}$ simplifies [4] to $\epsilon_{\mathrm{eff}}=\left(\epsilon_{1}+1\right) / 2$.

\section{Experiment}

The samples used in the present study were processed on a high electron mobility $\mathrm{GaAs} / \mathrm{Al}_{0.36} \mathrm{Ga}_{0.64} \mathrm{As}$ het-

\footnotetext{
* corresponding author; e-mail: marcin.bialek@fuw.edu.pl
}

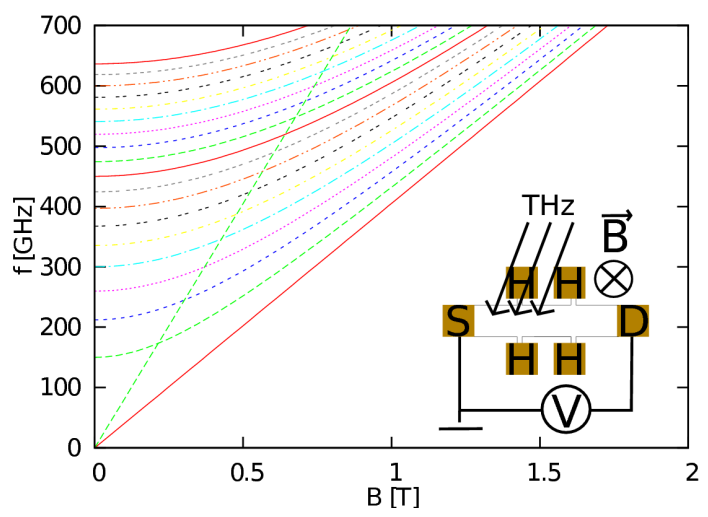

Fig. 1. Magnetoplasmon frequency as a function of the magnetic field for GaAs effective mass $m^{*}=0.0673 m_{\mathrm{e}}$, $f=\omega_{\mathrm{p}} / 2 \pi=150 \mathrm{GHz}$ and constant $\epsilon_{\mathrm{eff}}$. The lines show subsequent modes of the fundamental magnetoplasmon frequency. The straight green line shows the second harmonic of the cyclotron resonance (CR). Inset shows the electrical setup used for PV measurements and the configuration of the sample in the magnetic field. S, $\mathrm{D}$, and $\mathrm{H}$ denote the source, drain, and Hall voltage contact, respectively.

erostructure with an electron beam lithography. First, mesas (separating lines) were wet etched in order to define the basic shape of the samples. Second, metallic contacts were prepared by deposition of about $160 \mathrm{~nm}$ of $\mathrm{Au} / \mathrm{Ge} / \mathrm{Ni}$ alloy using evaporation and then heating the sample up to $430^{\circ} \mathrm{C}$ for a few min. The Hall-effect measurements at $4 \mathrm{~K}$ allowed us to estimate the electron concentration and mobility to be about $2.7 \times 10^{11} \mathrm{~cm}^{-2}$ and $6 \times 10^{5} \mathrm{~cm}^{2} /(\mathrm{Vs})$, respectively.

Each of three samples was supplied with two ohmic contacts used as current contacts (those will be called the source and the drain). Two of the samples were identical Hall bars, named with numbers 1 and 3. Four additional ohmic contacts at the sample edge, used as voltage probes in the Hall effect measurements, were 
disconnected (a floating potential) during photovoltage measurements. The third sample was a sample with the source and drain contacts only. The source-drain distance (length of the channel) in all three cases was $1300 \mu \mathrm{m}$, and the channel width was $65 \mu \mathrm{m}$ in the Hall bars and $80 \mu \mathrm{m}$ in the bar sample. Contacts were connected to sample holders with $25 \mu \mathrm{m}$ thick Au wire. All three samples were prepared on three separate pieces from the same wafer.

Samples were placed in a liquid helium cryostat at $4.2 \mathrm{~K}$. Magnetic field was produced by a superconducting coil. A $\mathrm{THz}$ radiation was led to the sample with an oversized waveguide of the diameter of $12 \mathrm{~mm}$. Two filters were used, one was a black polyethylene to filter visible and near-infrared light (energy higher than about $700 \mathrm{~cm}^{-1}$ ). This filter was placed just next to the sample to stop also far-infared radiation coming from warmer parts of the cryostat. The second filter was a white teflon to stop radiation in the range from $500 \mathrm{~cm}^{-1}$ to $1300 \mathrm{~cm}^{-1}$. THz beam was generated by an electronic source giving the frequency of $630-660 \mathrm{GHz}$ or 210-220 GHz. Usually, we measured a photovoltage (PV) signal as a function of a magnetic field for a constant radiation frequency. The PV signal was measured as a voltage between source and drain contacts, without any external bias of the sample. The source contact was grounded. A physical mechanism lying behind generation of the PV signal is rectification of oscillating currents induced in the electron plasma by the incoming radiation $[5,6]$. We have applied a phase-sensitive lock-in measurements system with an electronically modulated intensity of radiation. The modulation frequency was in the range from $13 \mathrm{~Hz}$ to $600 \mathrm{~Hz}$. When a Hall bar was used, the lateral voltage probes were disconnected (floating potential), and a PV signal was measured between the source and drain. Typically, the signal amplitude was between a few $\mu \mathrm{V}$ and $1 \mathrm{mV}$.

\section{Results}

The results obtained on one of two Hall bars are plotted as maps in Fig. 2 which shows a PV as a function of magnetic field in the frequency range 630-660 GHz. This picture clearly shows a series of up to sixteen parallel lines. The slope of these lines corresponds to the electron effective mass in GaAs, calculated from the CR frequency dependence on the magnetic field. The distance between the lines in the magnetic field is about $0.05 \mathrm{~T}$. The map for a $210-200 \mathrm{GHz}$ range (Fig. 3) shows a smaller number of lines more with a larger distance in magnetic field; clearly, one of these lines shows a different slope than the other. This is in a qualitative agreement with theoretical predictions shown in Fig. 1. For the $630-660 \mathrm{GHz}$ range we expect a series of almost parallel lines of the slope of the CR. One can also see the second harmonic of CR in 630-660 GHz results. In fields higher than CR field no pattern as clear as that is visible, although there are is some photovoltage signal in that range of $B$, contrary to theoretical predictions of detection spectra measured as a function of the magnetic field [7].

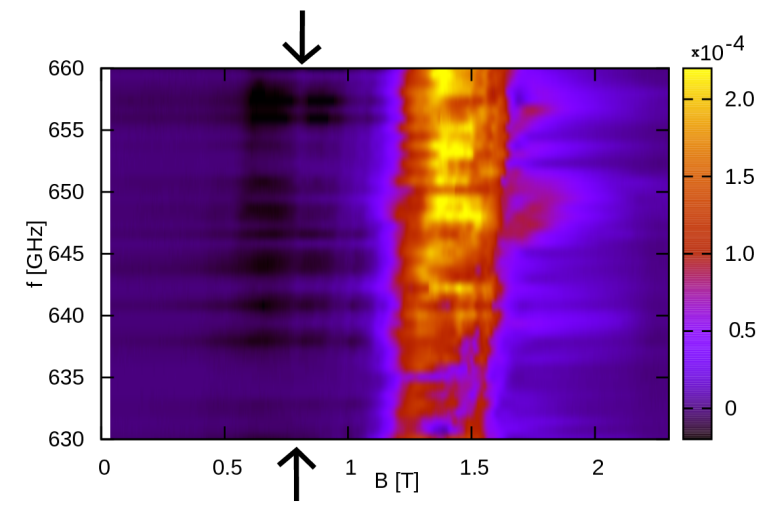

Fig. 2. Map of photovoltage in the $630-660 \mathrm{GHz}$ frequency range obtained with Hall bar 3. Arrows mark position of the CR second harmonic.

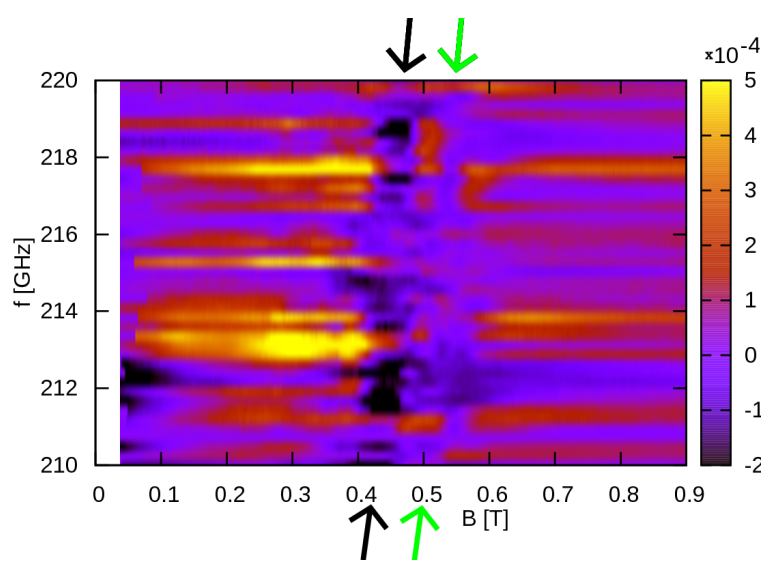

Fig. 3. Map of photovoltage in the $210-220 \mathrm{GHz}$ frequency range obtained with Hall bar 3. Arrows mark positions of two visible magnetoplasmon modes in this spectral range for Hall bar sample.

We have performed an analysis of the positions of the lines in magnetic field for chosen frequencies. With the resonance position in the magnetic field and the radiation frequency one can calculate plasmon frequency at zero magnetic field using Eq. (1). We assume that a wave vector of each mode is described by $k=n k_{0}$, where $n$ is the number of the mode, and $k_{0}$ is wave vector of the fundamental magnetoplasmon resonance (the line closest to the CR line). As a result we can plot a dispersion relation of observed magnetoplasmons as a function of the normalized wave vector $n=k / k_{0}$. This result is shown in Fig. 4. One can see that we obtain almost square root-like dependence. We fitted the experimental data with the theoretical dispersion relation. The numerical results of the fitting allows to calculate $k_{0}$ using Eq. (2).

Because the sample does not contain any periodic coupler, the only spacial boundary for the plasmons waves 


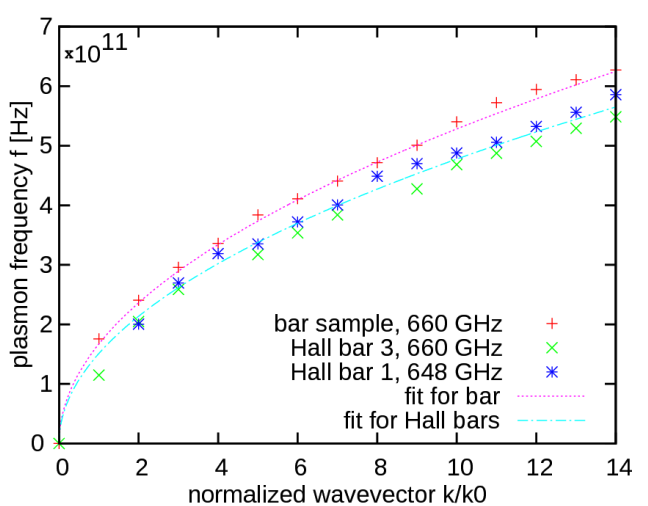

Fig. 4. Plasmon frequencies plotted vs. normalized wave vector $n=k / k_{0}$ (the mode number). The fitting gives the frequency of the basic magnetoplasmon mode equal to $151 \mathrm{GHz}$ for Hall bars (blue line) and $167 \mathrm{GHz}$ for the bar (red line).

are boundaries of the sample itself. Such a resonance in finite size samples is called depolarization shift [8]. Therefore, basic plasmons wave vector shall fit to the dimensions of the sample. Indeed, it is on the order of the width of the sample. When taking $\epsilon_{\mathrm{eff}}=7.0 \approx\left(\epsilon_{\mathrm{GaAs}}+1\right) / 2$ one obtains the value of the wavelength of the fundamental magnetoplasmon mode of about $64 \mu \mathrm{m}$ which is close to the width of the Hall bars $(65 \mu \mathrm{m})$. The result for the bar sample gives the length of the fundamental magnetoplasmon mode of $52 \mu \mathrm{m}$. Here the result does not fit to the channel width which is about $80 \mu \mathrm{m}$. This could be understood, however, as an excitation of three halves of the wavelength in the fundamental mode. The reasons for such a behaviour in this sample are not clear. Very similar coupling of the magnetoplasmon to the width of the Hall bar was observed previously in GaAs/AlGaAs heterostructures [4].
The observation of the second harmonic of the CR might be a result of the Bernstein modes in the sample [9]. The high number of observed modes of magnetoplasmons can be understood as a result of the retardation effects observed in large scale samples [8].

\section{Conclusion}

We have observed multiple magnetoplasmon modes in three samples of a macroscopic length processed on high quality GaAs/AlGaAs heterostructure. A dispersion relation of observed excitations is square root-like. Fitting a theoretical dispersion to the experimental data allows to determine the wavelength of the fundamental plasmon mode which is close to the sample width.

\section{Acknowledgments}

This work was partially supported by the Foundation for Polish Science grant POMOST/2010-1/8.

\section{References}

[1] F. Stern, Phys. Rev. Lett. 18, 546 (1967).

[2] E. Batke, Phys. Rev. B 34, 6951 (1986).

[3] N. Okisu, Appl. Phys. Lett. 48, 776 (1986).

[4] E. Vasiliadou, Phys. Rev. B 48, 148 (1993).

[5] M. Dyakonov, M. Shur, Phys. Rev. Lett. 71, 2465 (1993).

[6] M. Dyakonov, Semiconductors 42, 984 (2008).

[7] M.B. Lifshits, M.I. Dyakonov, Phys. Rev. B 80, 121304(R) (2009).

[8] S.A. Mikhailov, Phys. Rev. B 70, 165311 (2004).

[9] D.E. Bangert, Semicond. Sci. Technol. 11, 352 (1996). 